\title{
Reconstruction of Binary Matrices from Absorbed Projections
}

\author{
E. Balogh ${ }^{1}$, A. Kuba ${ }^{1}$, A. Del Lungo ${ }^{2}$, and M. Nivat ${ }^{3}$ \\ 1 Department of Applied Informatics, University of Szeged, \\ Árpád tér 2, H-6720 Szeged, Hungary \\ \{bmse, kuba\}@inf.u-szeged.hu \\ 2 Department of Mathematics, University of Siena, \\ Via del Capitano 15, 53100 Siena, Italy \\ dellungo@unisi.it \\ 3 Laboratoire d'Informatique Algorithmique: Fondements et Applications, \\ Université Paris 7 Denis-Diderot, Paris, France \\ tcsmn@club-internet.fr
}

\begin{abstract}
A generalization of the classical discrete tomography problem is considered: Reconstruct binary matrices from their absorbed row and column sums. We show that this reconstruction problem can be linked to a 3SAT problem if the absorption is characterized with the constant $\beta=\ln \left(\frac{1+\sqrt{5}}{2}\right)$.
\end{abstract}

Keywords: discrete tomography, reconstruction, absorption

\section{Introduction}

Let $A=\left(a_{i j}\right)_{m \times n}$ be a binary matrix and let be $\beta \geq 1$. Then we can define the absorbed row and column sums of $A R_{\beta}(A)$ and $S_{\beta}(A)$, respectively, as

$$
R_{\beta}(A)=R=\left(r_{1}, \ldots, r_{m}\right) \quad \text { where } \quad r_{i}=\sum_{j=1}^{n} a_{i j} \beta^{-j}, \quad i=1, \ldots, m,
$$

and

$$
S_{\beta}(A)=S=\left(s_{1}, \ldots, s_{n}\right) \quad \text { where } \quad s_{j}=\sum_{i=1}^{m} a_{i j} \beta^{-i}, \quad j=1, \ldots, n .
$$

Then the reconstruction problem of binary matrices with absorption knowing the projections along horizontal and vertical lines can be posed as

ReCONSTRUCTION $D A 2 D(\beta)$.

Instance: $\quad \beta \geq 1, m, n, R \in \mathbb{N}^{m}$, and $S \in \mathbb{N}^{n}$

Task: $\quad$ Construct a binary matrix $A$ with size $m \times n$ such that

$$
R_{\beta}(A)=R \quad \text { and } \quad S_{\beta}(A)=S .
$$

A. Braquelaire, J.-O. Lachaud, and A. Vialard (Eds.): DGCI 2002, LNCS 2301, pp. 392 4032002. (C) Springer-Verlag Berlin Heidelberg 2002 
If $\beta=1$ then we have the classical reconstruction problem of binary matrices without absorption (as summaries see e.g. [12]). Other $\beta$ values are suitable to describe the following model of the emission discrete tomography. Let us suppose that the discrete object represented by the binary matrix $A$ is in an absorbing material having absorption coefficient $\mu$. If we measure the horizontal and vertical projections of $A$, then we have the absorbed row and column sums, i.e., $R_{\beta}(A)$ and $S_{\beta}(A)$, where $\beta=e^{\mu}$. Some more explanation to the motivation of this problem see 34

Select, for example, the mathematically interesting case $\beta=\beta_{0}$ where

$$
\beta_{0}^{-1}=\beta_{0}^{-2}+\beta_{0}^{-3}
$$

giving a solution

$$
\beta_{0}=\frac{1+\sqrt{5}}{2}
$$

In this paper we discuss the problem of reconstruction of binary matrices from their row and column sums in the case of absorption characterized with $\beta_{0}$. A necessary and sufficient condition of uniqueness in this class is published in 34 .

In this paper we are going to connect this kind of reconstruction problem with 3SAT. The SAT and the different reconstruction problems have been connected already in 56.

\section{$2 \quad \boldsymbol{\beta}_{0}$-Representation}

Consider the row and column sums of the binary matrix $A$ in the case of $\beta=\beta_{0}$ :

$$
r_{i}=\sum_{j=1}^{n} a_{i j} \beta_{0}^{-j}, i=1, \ldots, m, \text { and } s_{j}=\sum_{i=1}^{m} a_{i j} \beta_{0}^{-i}, j=1, \ldots, n .
$$

Using the terminology of numeration systems we can say that the finite (binary) word $a_{i 1} \cdots a_{i n}$ is a (finite) representation in base $\beta_{0}$ (or a finite $\beta_{0}$ representation) of $r_{i}$ for each $i=1, \ldots, m$, and, similarly, $a_{1 j} \cdots a_{n j}$ is a $\beta_{0^{-}}$ representation of $s_{j}$ for each $j=1, \ldots, n$. The equations (6) mean also that the row and column sums of $A$ are nonnegative real numbers having a finite $\beta_{0^{-}}$ representation with $n$ and $m$ binary digits, respectively (including the eventually ending zeros).

Let $B_{k}$ denote the set of nonnegative real numbers having a $\beta_{0}$-representation with $k$ binary digits $(k>1)$, formally,

$$
B_{k}=\left\{\sum_{i=1}^{k} a_{i} \beta_{0}^{-i} \mid a_{i} \in\{0,1\}\right\}
$$

Then 


$$
r_{i} \in B_{n}, i=1, \ldots, m, \quad \text { and } \quad s_{j} \in B_{m}, j=1, \ldots, n,
$$

are necessary conditions for the existence of a matrix $A$ with

$$
R_{\beta_{0}}(A)=\left(r_{1}, \ldots, r_{m}\right) \quad \text { and } \quad S_{\beta_{0}}(A)=\left(s_{1}, \ldots, s_{n}\right) .
$$

\subsection{Switching in $\boldsymbol{\beta}_{0}$-Representations}

The $\beta_{0}$-representation is generally nonunique, because there are binary words with the same length representing the same number. For example, on the base of (4) it is easy to check the following equality between the 3 -digit-length $\beta_{0^{-}}$ representations

$$
100=011 .
$$

As direct consequences of (10), it is easy to see that

$$
\begin{aligned}
100 & =011 \\
10 x_{3} 00 & =01 x_{3} 11 \\
10 x_{3} 0 x_{5} 00 & =01 x_{3} 1 x_{5} 11 \\
10 x_{3} 0 x_{5} 0 x_{7} 00 & =01 x_{3} 1 x_{5} 1 x_{7} 11 \\
\ldots &
\end{aligned}
$$

where $x_{3}, x_{5}, x_{7}, \cdots$ denote the positions where both $\beta_{0}$-representations have the same (but otherwise arbitrary) binary digit. (That is, such kind of transformation $1(0 x)^{k-1} 00 \rightarrow 0(1 x)^{k-1} 11(k \geq 1)$ between the subwords of the $\beta_{0^{-}}$ representations can be performed without changing the represented value and without changing the values in the positions indicated by $x$ 's.) The transformations described by (10) and (11) are called switchings.

It is proved that any finite $\beta_{0}$-representation of a number can be get from its any other $\beta_{0}$-representation by switchings.

Lemma 1. [3] Let $a_{1} \cdots a_{k}$ and $b_{1} \cdots b_{k}$ be different, $k$-digit-length $\beta_{0}$ representations of the same number. Then $b_{1} \cdots b_{k}$ can be get from $a_{1} \cdots a_{k}$ by a finite number of switchings.

Consequence. If $a_{1} \cdots a_{k}$ and $b_{1} \cdots b_{k}$ are different, $k$-digit-length $\beta_{0}$-representations of the same number, then there are positions $i, i+1, i+2(1 \leq i \leq k-2)$ such that there is a switching between $a_{1} \cdots a_{k}$ and $b_{1} \cdots b_{k}$ on these positions.

\section{$2.2 \boldsymbol{\beta}_{0}$-Expansion}

The $k$-digit-length $\beta_{0}$-expansion is a particular $k$-digit-length $\beta_{0}$-representation that can be computed by the "greedy algorithm": Let $r \in B_{k}$, then its $\beta_{0^{-}}$ expansion $a_{1} \cdots a_{k}$ is determined as

$$
\begin{aligned}
& r_{0}:=r, \\
& a_{i}:=\left\lfloor\beta_{0} \cdot r_{i-1}\right\rfloor, \quad r_{i}:=\left\{\beta_{0} \cdot r_{i-1}\right\}, \quad i=1, \ldots, k,
\end{aligned}
$$


where $\lfloor$.$\rfloor and \{$.$\} denote the integer and fractional, respectively, part of the$ argument. It is clear that the $k$-digit-length $\beta_{0}$-expansion of any number $r \in B_{k}$ is uniquely determined (it is not the case with the $k$-digit-length $\beta_{0}$-representations as we saw it in the previous subsection).

The finite $\beta_{0}$-expansion is characterized by the following property

Proposition 1. [3] Let $a_{1}, \ldots, a_{k} \in\{0,1\} \quad(k \geq 1)$. The word $a_{1} \cdots a_{k}$ is the $\beta_{0}$-expansion of a number $r \in B_{k}$ if and only if it has the form

$$
a_{1} \cdots a_{k}=T U V, \text { where } T=0 \cdots 0, \quad T=1 \cdots 1, \quad \text { or } \quad T=\lambda,
$$

( $\lambda$ denotes the empty symbol),

$$
U=U_{1} \cdots U_{u}, \quad u \geq 0, \quad \text { such that } \quad U_{i}=10 \cdots 0, \quad i=1, \ldots, u,
$$

and each $U_{i}$ contains at least one 0 ,

$$
V=1 \quad \text { or } \quad V=\lambda
$$

and at least one of $T, U$, and $V$ is not the empty symbol $\lambda$.

\section{$3 \quad \beta_{0}$-Representation and 3SAT Clauses}

We are going to describe the $\beta_{0}$-representation by 3 SAT expressions, that is, by Boolean expressions in conjunctive normal form with at most three literals in each clause. Let $r$ be a real number having a $k$-digit long $\beta_{0}$-representation, $a_{1} \cdots a_{k}$. Let $z_{1}, \ldots, z_{k}$ be Boolean variables and $L$ be a Boolean function of $z_{1}, \ldots, z_{k}$, that is, $L=L\left(z_{1}, \ldots, z_{k}\right)$. We say that the Boolean values $a_{1}, \ldots, a_{k}$ satisfy $L$ if $L\left(z_{1}=a_{1}, \ldots, z_{k}=a_{k}\right)$ is true.

Now we are going to give the set of clauses, denoted by $K$, by which all $k$-digit length $\beta_{0}$-representations of any $r \in B_{k}$ can be described for any $k>1$. Let $a_{1} \cdots a_{k}$ the $k$-digit-length $\beta_{0}$-expansion of $r$. Then, by Proposition 1

$$
a_{1} \cdots a_{k}=T U V
$$

where $T, U$, and $V$ are given by (13), and (14), respectively. Accordingly,

$$
K=T T \cup U U \cup V V
$$

where $T T, U U$, and $V V$ denote the subsets of clauses describing the corresponding parts $T, U$, and $V$.

First, consider the non-constant part of the $\beta_{0}$-representations, $U=$ $U_{1} \cdots U_{u}(u \geq 0)$. On the base of Lemma 1 we know that all $\beta_{0}$-representations of any $r \in B_{k}$ can be generated from the $\beta_{0}$-expansion of $r$ by elementary switchings. Accordingly, the clauses $U U$ have to describe the set of binary words generated from $U_{k}$ by elementary switchings (see Fig. 11). The elementary switchings done in $U$ can be classified into two classes according to the places of switchings: 
(i) The switchings done in the positions of one $U_{i}$. (ii) The switchings done in the positions of $U_{i}$ and $U_{i+1}$, i.e. the last 1 of $U_{i}$ "overflows" into the first position of $U_{i+1}$ as a consequence of switchings. There can be such a switching if the length of $U_{i}$ is even and the length of $U_{i+1}$ is not less than 3 (see the $\beta_{0}$-representations in Fig. 11indicated by arrows).

There are two consequences of overflowing switchings: We have different clauses for $U_{i}$ having even or odd length $l_{i}$ and the sets of clauses of $U_{i}, i=$ $1, \cdots u$, are not completely independent.

The clauses of $U U$ are given with the help of the Boolean variables $\gamma_{j}, \delta_{j}, \varphi_{j}, \psi_{j}$, and $\chi_{j}, j=w_{1}, w_{1}+1, \cdots, w_{u}+l_{u}-1$, i.e. for all the variables of $U U$. For each $j$ exactly one of these variables has value 1 (see the clauses of POSITIONS later). For this reason each binary word satisfying the clauses described by these variables can be represented in a 1-to-1 correspondence by a word of the alphabet $\{\gamma, \delta, \varphi, \psi, \chi\}$, indicating which variable has value 1 on that position. For example, $z_{1} z_{2} z_{3}=\psi \gamma \delta$ means that $\gamma_{1}=0, \gamma_{2}=1, \gamma_{3}=0$, $\delta_{1}=0, \delta_{2}=0, \delta_{3}=1, \varphi_{1}=0, \varphi_{2}=0, \varphi_{3}=0, \psi_{1}=1, \psi_{2}=0, \psi_{3}=0$, $\chi_{1}=0, \chi_{2}=0, \chi_{3}=0$. The variables $\gamma_{j}, \delta_{j}, \varphi_{j}, \psi_{j}$, and $\chi_{j}$ describing the clauses of $U U$ will be transformed to 0's and 1's as follows:

$$
\varphi_{j} \Rightarrow a_{j}=0, \delta_{j} \Rightarrow a_{j}=1, \psi_{j} \Rightarrow a_{j}=0, \gamma_{j} \Rightarrow a_{j}=1, \chi_{j} \Rightarrow a_{j}=1 .
$$

Continuing the previous example, then $\psi \gamma \delta=011$.

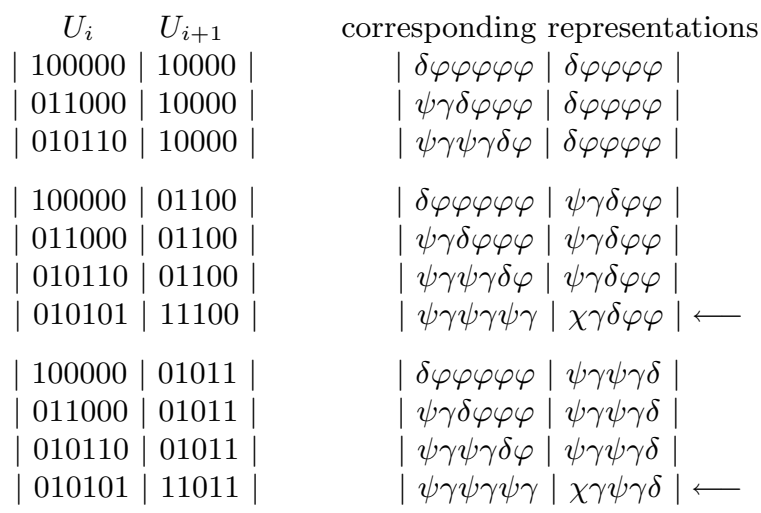

Fig. 1. All $\beta_{0}$-representations of $U_{i} U_{i+1}$ generated by elementary switchings and the corresponding representations with the variables $\gamma, \delta, \varphi, \psi$, and $\chi$ (when $l_{i}=6$ and $\left.l_{i+1}=5\right)$. The positions of $U_{i}$ and $U_{i+1}$ are separated by vertical lines. The "overflowing" 1 's are indicated by $\chi$ in the rows with arrows.

Let $B\left(U_{i}\right)$ denote the set of (binary) sequences of $U_{i}$. Clearly,

$$
B\left(U_{i}\right)=\{01\}^{b} 1\{0\}^{c},
$$


where $b$ and $c$ are nonnegative integers such that $b+1+c=l_{i}$. Then the binary sequences of $U_{i} U_{i+1}, B\left(U_{i} U_{i+1}\right)$, can be given as

$$
B\left(U_{i} U_{i+1}\right)= \begin{cases}B\left(U_{i}\right) B\left(U_{i+1}\right), & \text { if } l_{i} \text { is odd } \\ B\left(U_{i}\right) B\left(U_{i+1}\right) \cup\{01\}^{l_{i} / 2} 1 B^{(0)}\left(U_{i+1}\right), & \text { if } l_{i} \text { is even }\end{cases}
$$

where $B^{(0)}\left(U_{i+1}\right)$ denotes the set of subsequences created from those sequences of $B\left(U_{i+1}\right)$, where the first element is 0 , by omitting just this first 0 . For example, if $l_{i}=6$ and $l_{i+1}=5$ then $B\left(U_{i}\right)=\{100000,011000,010110\}, B\left(U_{i+1}\right)=$ $\{10000,01100,01011\}$, and $B^{(0)}\left(U_{i+1}\right)=\{1100,1011\}$.

We can describe these sequences with the letters $\gamma, \delta, \varphi, \psi$, and $\chi$ as follows. Corresponding to (18) and (19)

$$
\begin{gathered}
B\left(U_{i}\right)=\{\psi \gamma\}^{a} \delta\{\varphi\}^{b}, \\
B\left(U_{i} U_{i+1}\right)= \begin{cases}B\left(U_{i}\right) B\left(U_{i+1}\right), & \text { if } l_{i} \text { is odd } \\
B\left(U_{i}\right) B\left(U_{i+1}\right) \cup\{\psi \gamma\}^{l_{i} / 2} \chi B^{(\psi)}\left(U_{i+1}\right), & \text { if } l_{i} \text { is even. }\end{cases}
\end{gathered}
$$

According to (17) $\psi, \varphi$ denote $0, \gamma, \delta$, and $\chi$ denote $1 . B$ and $B^{(\psi)}$ are defined in these sequences analogously to (18) and (19). Examples of generated in this way and the corresponding $\beta_{0}$-representations are in Fig. 1

The following sets of clauses will define a subword $U_{i}$.

$$
\begin{gathered}
\operatorname{DELTA}=\bigwedge_{j=w_{i}}^{w_{i}+l_{i}-2}\left(\delta_{j} \Rightarrow \varphi_{j+1}\right) \wedge \bigwedge_{j=w_{i}+1}^{w_{i}+l_{i}-1}\left(\delta_{j} \Rightarrow \gamma_{j-1}\right) \wedge \\
\bigwedge_{j=1}^{\left[\frac{l_{i}}{2}\right]} \overline{\delta_{w_{i}+2 j-1}} \wedge\left(\varphi_{w_{i}+1} \Rightarrow \delta_{w_{i}}\right) .
\end{gathered}
$$

The position of $\delta$ is crucial, because knowing this position all the elements succeeding $\delta$ can be computed as it is described in the first part of this rule and all elements preceding $\delta$ can be computed as it is described in the second part. $\delta$ cannot be on an even position in the subword $U_{i}$. The last part of DELTA expresses that if there is a $\varphi$ in the second position then there is a $\delta$ in the first one.

$$
P H I=\bigwedge_{j=w_{i}+1}^{w_{i}+l_{i}-2}\left(\varphi_{j} \Rightarrow \varphi_{j+1}\right) \wedge \overline{\varphi_{w_{i}}} .
$$

In other words, $\varphi$ can be followed only by $\varphi$ and $\varphi$ cannot stand on the first position of the subword.

$$
\text { GAMMAPSI }=\bigwedge_{j=w_{i}+2}^{w_{i}+l_{i}-1}\left(\gamma_{j} \Rightarrow \psi_{j-1}\right) \wedge \bigwedge_{j=w_{i}}^{w_{i}+l_{i}-2}\left(\psi_{j} \Rightarrow \gamma_{j+1}\right)
$$


The only predecessor of $\gamma$ is $\psi$ and the only successor of $\psi$ is $\gamma$.

$$
C H I=\bigwedge_{j=w_{i}+1}^{w_{i}+l_{i}-1} \overline{\chi_{j}} \wedge\left(\chi_{w_{i}} \Rightarrow \gamma_{w_{i}+1}\right)
$$

$\chi$ can stand only on the first position.

$$
G A M M A=\bigwedge_{j=w_{i}}^{w_{i}+l_{i}-1}\left(\gamma_{j} \Rightarrow \overline{\varphi_{j+1}}\right)
$$

$\gamma$ cannot be followed by $\varphi$.

$$
\begin{aligned}
\operatorname{POSITIONS}= & \bigwedge_{j=1}^{\left[\frac{l_{i}}{2}\right]}\left(\varphi_{w_{i}+2 j} \vee \gamma_{w_{i}+2 j}\right) \wedge\left(\delta_{w_{i}} \vee \psi_{w_{i}} \vee \chi_{w_{i}}\right) \wedge \\
& \bigwedge_{j=1}^{\left[\frac{l_{i}}{2}\right]}\left(\delta_{w_{i+2 j-1}} \vee \psi_{w_{i+2 j-1}} \vee \varphi_{w_{i+2 j-1}}\right) .
\end{aligned}
$$

On an even position in a subword can be $\varphi$ or $\gamma$, on the first position in the subword can stand $\delta, \psi$, or $\chi$, and on odd positions in the subword can stand $\delta$, $\psi$, or $\varphi$. These are the only clauses containing 3 variables.

$$
E V E N=\left(\gamma_{w_{i}+l_{i}-1} \Rightarrow \chi_{w_{i}+l_{i}}\right)
$$

Actually $w_{i}+l_{i}=w_{i+1}$, the first element of the subword $U_{i+1}$. This means that a subword with even length can influence the next subword. In this case the first element is a $\chi$ followed by $\gamma$.

$$
O D D=\overline{\chi_{w_{i}+l_{i}}} \wedge \overline{\psi_{w_{i}+l_{i}-1}}
$$

A subword with odd length cannot influence the next subword, this means that the first element of the next subword cannot be $\chi$ and the last element cannot be $\psi$.

$$
D I S J=\bigwedge_{j=1}^{l_{i}}\left(A_{j} \Rightarrow \overline{B_{j}}\right), \text { for symbols } A, B \in\{\varphi, \psi, \gamma, \delta, \chi\}, \text { where } A \neq B
$$

The clauses mean that exactly one of the variables $\varphi, \psi, \gamma, \delta$, and $\chi$ has the value 1 , for each $j=1, \cdots, l_{i}$. 
The clauses for a subword $\boldsymbol{U}_{\boldsymbol{i}}$. Knowing the length of the subword $U_{i}$ we can construct a corresponding 3SAT expression:

$$
K_{i}=\left\{\begin{array}{l}
D E L T A \wedge P H I \wedge G A M M A P S I \wedge C H I \\
\wedge G A M M A \wedge P O S I T I O N S \wedge O D D, \quad \text { if } l_{i} \text { is odd } \\
D E L T A \wedge P H I \wedge G A M M A P S I \wedge C H I \\
\wedge G A M M A \wedge P O S I T I O N S \wedge E V E N, \quad \text { if } l_{i} \text { is even }
\end{array}\right.
$$

The clauses describing $\boldsymbol{U} \boldsymbol{U}$. Let $\Gamma=\left(\gamma_{1}, \cdots, \gamma_{k}\right), \Delta=\left(\delta_{1}, \cdots, \delta_{k}\right), \Phi=$ $\left(\varphi_{1}, \cdots, \varphi_{k}\right), \Psi=\left(\psi_{1}, \cdots, \psi_{k}\right)$, and $X=\left(\chi_{1}, \cdots, \chi_{k}\right)$ be the vectors of Boolean variables. Then $U U=U U(r ; \Gamma, \Delta, \Phi, \psi, X)$ is defined as follows:

$$
U U=\bigwedge_{i=1}^{u} K_{i} .
$$

The clauses describing $\boldsymbol{T T}$ and $\boldsymbol{V} \boldsymbol{V}$. In these clauses the same variables are as used in $U U$. Since the subwords corresponding to $T$ and $V$ have constant values in each $\beta_{0}$-representation of the same $r \in B_{k}$, the clauses describing these parts are

$$
T T= \begin{cases}\gamma_{1}=\cdots=\gamma_{l_{t}}=0, \delta_{1}=\cdots=\delta_{l_{t}}=0, & \\ \varphi_{1}=\cdots=\varphi_{l_{t}}=0, \psi_{1}=\cdots=\psi_{l_{t}}=0, & \text { if } T=0 \cdots 0 ; \\ \chi_{1}=\cdots=\chi_{l_{t}}=1, & \\ \gamma_{1}=\cdots=\gamma_{l_{t}}=0, \delta_{1}=\cdots=\delta_{l_{t}}=1, & \\ \varphi_{1}=\cdots=\varphi_{l_{t}}=0, \psi_{1}=\cdots=\psi_{l_{t}}=0, & \text { if } T=1 \cdots 1 ; \\ \chi_{1}=\cdots=\chi_{l_{t}}=0, & \text { if } T=\lambda, \\ \phi, & \end{cases}
$$

and

$$
V V= \begin{cases}\gamma_{k}=0, \delta_{k}=1, \varphi_{k}=0, \psi_{k}=0, \chi_{k}=0, & \text { if } V=1 \\ \phi, & \text { if } V=\lambda\end{cases}
$$

The clauses describing $K$. As we saw $T T, U U, V V$, and so $K$ are defined with the help of $r, \Gamma, \Delta, \Phi, \Psi$, and $X$, i.e.,

$$
K=K(r ; \Gamma, \Delta, \Phi, \Psi, X) .
$$

$K$ is given by (16) explicitly. 
Theorem 1. Let $r \in B_{k}$ and $a_{1}, \ldots, a_{k}$ be a binary word. $a_{1}, \ldots, a_{k}$ is a $\beta_{0}$-representations of $r$ if and only if there are vectors $\Gamma, \Delta, \Phi, \Psi$, and $X$ of Boolean values such that $a_{1}, \ldots, a_{k}$ is transformed by these vectors by (17) and $K(r ; \Gamma, \Delta, \Phi, \Psi, X)$ is true.

Proof. Let $a_{1} \cdots a_{k}$ be a $k$-digit-length $\beta_{0}$-representation of $r$. The corresponding word of $\gamma, \delta, \varphi, \psi$, and $\chi$ is uniquely determined on the base of the forms (20) and (21). It is easy to check that all clauses of $K$ (i.e. TT, $V V, D E L T A, \cdots$, $D I S J)$ are satisfied by any word given by (20) and (21).

In order to prove the other direction, consider an arbitrary word $W$ satisfying the clauses of $K$. $W$ has the uniquely determined structure $T U V$, where $T$ same as (13), $V$ same as (15) and $U$ is a word of $\gamma, \psi, \varphi, \delta$, and, $\chi$. We have to show that $U$ is a sequence of subsequences $U_{i}$, each of them satisfying (20) and (21). Knowing $r$ we can determine the lengths $l_{i}$ and positions of all $U_{i}, i=1, \cdots u$.

Now we identify the subsequence $U_{i}$ with length $l_{i}$ starting from the end of $U$.

1. $l_{i}$ is odd. According to POSITIONS, in the $l_{i}$ th position can be $\delta, \psi$, or $\varphi$.

a. In the $l_{i}$ position there is a $\delta$. Now we have to prove that before $\delta$ there are only pairs of $\psi \gamma$. From DELTA it follows that in the position $l_{i}-1$ there is a $\gamma$. Let $\gamma$ the position $2 j$, before $\delta$. From GAMMAPSI it follows that in the position $2 j-1$ there is a $\psi$. From POSITIONS it follows that in the position $2 j-2$ there can be $\varphi$ or $\gamma$. If in the position $2 j-2$ is a $\varphi$, then according to $P H I$ in the position $2 j-1$ should be $\varphi$ which is a contradiction (from $D I S J$ ). This means, that in the position $2 j-2$ is a $\gamma$, and let $j=j-1$. This step has to be repeated till $j>1$. If $j=1$, i.e. in the second position is $\gamma$, then from POSITIONS we have that in the first position can be $\delta, \psi$, or $\chi$. If in the first position is $\delta$ then from DELTA follows that in the second position should be $\varphi$ which is a contradiction. Conform to the equations (20) and (21), in the first position can be $\psi$ or $\chi$, in this last case there is an overflow.

b. In the $l_{i}$ position there is a $\psi$ This in contradiction with $O D D$.

c. In the $l_{i}$ position there is a $\varphi$. From POSITIONS it follows that in the previous position can be $\varphi$ or $\gamma$. If it is $\gamma$, then from GAMMA it follows that in the $l_{i}$ th position cannot be $\varphi$ which is a contradiction. This means, that in the position $l_{i}-1$ is $\varphi$. If $l_{i}-2=1$ then in this position is $\delta$ (from DELTA). If $l_{i}-2>1$ then from POSITIONS it follows that in the position $l_{i}-2$ can be $\delta, \psi$, or $\varphi$. If in the position $i-2$ is $\delta$ then similar to Case a. we can prove that $U_{i}$ satisfies (20) and (21). If in the position $l_{i}-2$ is $\varphi$ then similar to Case c. we can prove that $U_{i}$ satisfies (20) and (21). If in the position $l_{i}-2$ is $\psi$ then from GAMMAPSI follows that in the position $l_{i}-1$ is $\gamma$ and this is in contradiction with $D I S J$.

2. $l_{i}$ is even. According to POSITIONS in the position $l_{i}$ can be $\varphi$ or $\gamma$. If it is $\varphi$ then using a similar deduction as in Case C. we can prove that $U_{i}$ satisfies (20) and (21). If in the position $l_{i}$ is $\gamma$, then conform $E V E N$ in the 
next position is $\chi$ and conform $C H I$ in the position $l_{i}+2$ is $\gamma$, which means that $U_{i}$ satisfies (20) and (21).

\section{The Reconstruction Algorithm}

In order to solve the reconstruction problem $D A 2 D\left(\beta_{0}\right)$ we express the $\beta_{0^{-}}$ representations of the absorbed row and column sums with 3SAT clauses. Boolean variables $\Gamma^{(h)}=\left(\gamma_{i j}^{(h)}\right)_{m \times n}, \Delta^{(h)}=\left(\delta_{i j}^{(h)}\right)_{m \times n}, \Phi^{(h)}=\left(\varphi_{i j}^{(h)}\right)_{m \times n}$, $\Psi^{(h)}=\left(\psi_{i j}^{(h)}\right)_{m \times n}$, and $X^{(h)}=\left(\chi_{i j}^{(h)}\right)_{m \times n}$ are for describing relations of column sums ( $h$ stands for horizontal), and $\Gamma^{(v)}=\left(\gamma_{i j}^{(v)}\right)_{m \times n}, \Delta^{(v)}=\left(\delta_{i j}^{(v)}\right)_{m \times n}, \Phi^{(v)}=$ $\left(\varphi_{i j}^{(v)}\right)_{m \times n}, \Psi^{(v)}=\left(\psi_{i j}^{(v)}\right)_{m \times n}$, and $X^{(v)}=\left(\chi_{i j}^{(v)}\right)_{m \times n}$ for describing relations of column sums ( $v$ stands for vertical), Let, furthermore, $\left.\Gamma_{i \cdot}^{(h)}=\left(\gamma_{i 1}^{(h)}\right), \cdots, \gamma_{i n}^{(h)}\right)$ be the $i$ th row of $\Gamma^{(h)}, i=1, \cdots, m$ and $\left.\Gamma_{\cdot j}^{(v)}=\left(\gamma_{1 j}^{(v)}\right), \cdots, \gamma_{m j}^{(V)}\right)^{T}$ be the $j$ th column of $\Gamma^{(v)}, j=1, \cdots, n . \Delta_{i \cdot}^{(h)}, \Phi_{i \cdot}^{(h)}, \Psi_{i \cdot}^{(h)}, X_{i \cdot}^{(h)}, \Delta_{\cdot j}^{(v)}, \Phi_{\cdot j}^{(v)}, \Psi_{\cdot j}^{(v)}$, and $X_{\cdot j}^{(v)}$, be defined similarly.

The clauses describing the rows and columns. Now we can describe a whole row of the discrete set to be reconstructed by the following subset of clauses:

$$
K^{(h)}\left(r_{i} ; \Gamma_{i .}^{(h)}, \Delta_{i .}^{(h)}, \Phi_{i .}^{(h)}, \Psi_{i .}^{(h)}, X_{i .}^{(h)}\right)=T T \wedge U U \wedge V V, i=1, \cdots, m,
$$

where $T T, U U$, and $V V$ are defined in the previous section. All clauses describing the absorbed row sums are given by

$$
\begin{aligned}
L^{(h)} & =L^{(h)}\left(R, \Gamma^{(h)}, \Delta^{(h)}, \Phi^{(h)}, \Psi^{(h)}, X^{(h)}\right) \\
& =\bigwedge_{i=1}^{m} K^{(h)}\left(r_{i} ; \Gamma_{i \cdot}^{(h)}, \Delta_{i \cdot}^{(h)}, \Phi_{i \cdot}^{(h)}, \Psi_{i \cdot}^{(h)}, X_{i \cdot}^{(h)}\right) .
\end{aligned}
$$

Similarly, the columns can be described by

$$
K^{(v)}\left(s_{j} ; \Gamma_{\cdot j}^{(v)}, \Delta_{\cdot j}^{(v)}, \Phi_{\cdot j}^{(v)}, \Psi_{\cdot j}^{(v)}, X_{\cdot j}^{(v)}\right)=T T \wedge U U \wedge V V, j=1, \cdots, n
$$

and

$$
\begin{aligned}
L^{(v)} & =L^{(v)}\left(R, \Gamma^{(v)}, \Delta^{(v)}, \Phi^{(v)}, \Psi^{(v)}, X^{(v)}\right) \\
& =\bigwedge_{i=1}^{m} K^{(v)}\left(s_{j} ; \Gamma_{\cdot j}^{(v)}, \Delta_{\cdot j}^{(v)}, \Phi_{\cdot j}^{(v)}, \Psi_{\cdot j}^{(v)}, X_{\cdot j}^{(v)}\right) .
\end{aligned}
$$


The clauses describing the binary matrix. The last step is to define the connections between the Boolean matrices

$$
\begin{aligned}
C O N N & =\left(\bigwedge_{i 1, j 1} \varphi_{i 1, j 1}^{(h)} \Rightarrow \overline{\gamma_{i 1, j 1}^{(v)}}\right) \wedge\left(\bigwedge_{i 1, j 1} \varphi_{i 1, j 1}^{(h)} \Rightarrow \overline{\delta_{i 1, j 1}^{(v)}}\right) \wedge\left(\bigwedge_{i 1, j 1} \varphi_{i 1, j 1}^{(h)} \Rightarrow \overline{\chi_{i 1, j 1}^{(v)}}\right) \wedge \\
& \wedge\left(\bigwedge_{i 1, j 1} \psi_{i 1, j 1}^{(h)} \Rightarrow \overline{\gamma_{i 1, j 1}^{(v)}}\right) \wedge\left(\bigwedge_{i 1, j 1} \psi_{i 1, j 1}^{(h)} \Rightarrow \overline{\delta_{i 1, j 1}^{(v)}}\right) \wedge\left(\bigwedge_{i 1, j 1} \psi_{i 1, j 1}^{(h)} \Rightarrow \overline{\chi_{i 1, j 1}^{(v)}}\right) \wedge \\
& \wedge\left(\bigwedge_{i 1, j 1} \gamma_{i 1, j 1}^{(h)} \Rightarrow \overline{\varphi_{i 1, j 1}^{(v)}}\right) \wedge\left(\bigwedge_{i 1, j 1} \gamma_{i 1, j 1}^{(h)} \Rightarrow \overline{\psi_{i 1, j 1}^{(v)}}\right) \wedge\left(\bigwedge_{i 1, j 1} \delta_{i 1, j 1}^{(h)} \Rightarrow \overline{\varphi_{i 1, j 1}^{(v)}}\right) \wedge \\
& \wedge\left(\bigwedge_{i 1, j 1} \delta_{i 1, j 1}^{(h)} \Rightarrow \overline{\psi_{i 1, j 1}^{(v)}}\right) \wedge\left(\bigwedge_{i 1, j 1} \chi_{i 1, j 1}^{(h)} \Rightarrow \overline{\varphi_{i 1, j 1}^{(v)}}\right) \wedge\left(\bigwedge_{i 1, j 1} \chi_{i 1, j 1}^{(h)} \Rightarrow \overline{\psi_{i 1, j 1}^{(v)}}\right)
\end{aligned}
$$

The 3SAT expression describing the whole discrete set is:

$$
L^{(h)} \wedge L^{(v)} \wedge C O N N .
$$

That is, in order to solve the reconstruction problem $D A 2 D\left(\beta_{0}\right)$ we have to do the following steps:

1. Determine the $\beta_{0}$-expansions of $r_{i}, i=1, \cdots, m$, and $j=1, \cdots n$.

2. On the base of $\beta_{0}$-expansions give the 3SAT expression (27).

3. Solve the 3SAT problem using an efficient SAT solver (e.g. CSAT, see [7]).

4. If there is a solution of the 3SAT problem, give the binary matrix solution on the base of (17).

\section{Discussion}

A method is given to solve the reconstruction problem $D A 2 D\left(\beta_{0}\right)$, i.e., to reconstruct a binary matrix from it absorbed row and column sums, when the absorption can be represented by the special value $\beta_{0}$. It is shown that the problem $D A 2 D\left(\beta_{0}\right)$ can be transformed to a 3SAT expression such that if there is a solution of the 3SAT expression then it gives also a solution of the reconstruction problem (see Section 4).

It is a natural question that how this method can be extended to other values of $\beta$. We believe that this idea is specific and cannot be generalised directly to all possible values of $\beta$. However, it is relative easy to show that very similar results are true for $\beta$ 's having the property

$$
\beta^{-1}=\beta^{-2}+\beta^{-3}+\cdots+\beta^{-l},
$$

where $l \geq 3$. Then the switchings can be described by similar relations as in (11), $\beta$-representations can be given similarly as in Section 3, and so the reconstruction problem can be reduced to a $3 \mathrm{SAT}$ problem in such cases. 
Acknowledgements. This work was supported by the grant OTKA T 032241.

\section{References}

1. Brualdi, R.A.: Matrices of zeros and ones with fixed row and column sums. Linear Algebra and Its Applications 33 (1980) 159-231.

2. Herman, G.T., Kuba, A. (Eds.): Discrete Tomography: Foundations, Algorithms and Applications. Birkhäuser, Boston (1999).

3. Kuba, A., Nivat, M.: A Sufficient condition for non-uniqueness in binary tomography with absorption, Technical Report, University of Szeged (2001).

4. Kuba, A., Nivat, M.: Reconstruction of discrete sets with absorption, accepted for publication in Linear Algebra and its Applications (2001).

5. M. Chrobak, C. Dürr, Reconstructing hv-convex polyominoes from orthogonal projections, Information Processing Letters 69 (1999) 283-289.

6. Y. Boufkhad, O. Dubois, and M. Nivat, Reconstructing (h,v)-convex twodimensional patterns of objects from approximate horizontal and vertical projections, to appear in Theoretical Computer Science.

7. O. Dubois, P. André, Y. Boufkhad, and J. Carlier, SAT versus UNSAT, in Second DIMACS Implementation Challenge, D. Johnson and M. A. Trick, eds., DIMACS Series in Discrete Mathematics and Theoretical Computer Science, AMS, 1993. 